\title{
The parameter estimation of Hyperbolic Modulation Frequency Signal based on cascade atom dictionary
}

\author{
LIN Congren ${ }^{1, a}$, YUAN Yuting ${ }^{1, b}$, LU Xiaoying ${ }^{1}$, SUN Haixin $^{1}$ \\ ${ }^{1}$ College of information science and technology, Xiamen University, Xiamen, China \\ ayuan15033091629@163.com, ${ }^{\mathrm{b}} 18106980839 @ 163 . c 0 m$ \\ Corresponding author: lincongren@163.com
}

Keywords: cascade atom dictionary, HFM signal, parameter estimation.

\begin{abstract}
As a kind of non-stationary time-frequency signal, Hyperbolic Modulation Frequency is widely used in the field of underwater communication, radar and wireless communications. Especially under the condition of the strong doppler effect, the doppler resistance of HFM signal is better than that of linear frequency modulation signal. In this article, in view of the HFM signals, a new method is proposed that the parameters of the HFM signal are detected by using the established cascade atom dictionary. The adjustable frequency of HFM signals is detected through establishing time scale atomic dictionary. Besides, the initial frequency is also detected by establishing time-frequency atom dictionary of a cascade form. At last, the computer simulation and pool test confirm that the parameter estimation of HFM signal based on cascade atom library method is reasonable.
\end{abstract}

\section{Introduction}

Due to the characteristics of time varying frequency, low probability of intercept technology, non-stationary signal is widely used in the field of radar, sonar, and wireless communications. Linear frequency modulation (LFM) signal and nonlinear frequency modulation (NLFM) signal are two typical non-stationary signals, and its parameter estimation method is the hotspot in the field of communication. As LFM signal has significant convergent characteristic through the fractional Fourier transform, Fractional Fourier transform has been widely used in the OFDM system as one of the main technical means of LFM signal parameter estimation [1]. The HFM has better tolerance in target moving and its straight line property of ridge is more convenient for signal processing. These advantages made HFM signals widely used in the radar, wireless communications and underwater communications. What's more, the HFM signal itself was been deep researched [3]. The literature [4] detected the HFM signal by the method of Wavelet - Radon transform. The result was relatively accurate ignoring of the relatively large amount of calculation. Literature [5] detected the HFM signal through the calculation of instantaneous frequency of the signal, but the concrete process did not founded. Literature [6] proved that the instantaneous frequency of HFM signal was a linear function after self-CWT ridge. The parameters of HFM signals can be estimated by establishing a cascade atom dictionary.

\section{HFM signal}

Considering a HFM signal with rectangle envelop,

$$
\begin{aligned}
& \mu(\mathrm{t})=\frac{1}{\sqrt{\mathrm{T}}} \operatorname{rect}\left(\frac{\mathrm{t}}{\mathrm{T}}\right) * \exp \left\{-\mathrm{j}\left[2 \pi \mathrm{K} \ln \left(1-\frac{\mathrm{t}}{\mathrm{t}_{0}}\right)\right]\right\} \\
& \mathrm{f} 1(\mathrm{t})=\frac{\mathrm{K}}{\mathrm{t}_{0}-\mathrm{t}}
\end{aligned}
$$

$\mathrm{K}=\frac{\mathrm{Tf}_{\max } \mathrm{f}_{\min }}{\mathrm{B}} ; \mathrm{t}_{0}=\frac{\mathrm{f}_{0} \mathrm{~T}}{\mathrm{~B}}, \mathrm{f}_{\text {max }}, \mathrm{f}_{\min }$ are minimum and maximum frequency respectively, $\mathrm{f}_{0}$ is the algorithm frequency. 


\section{Cascade dictionary}

The single atom dictionary commonly used for sparse decomposition will be introduced before establishing a cascade atom dictionary.

(1) The frequency atoms. Such as cosine atoms composed of cosine matrix.

(2) The time scale atoms. These atoms are made by wavelet discrete atoms.

(3) The time-frequency atom. It is usually made up through all kinds of window functions which are modulated.

In this paper the cascade atom dictionary is made up of time scales atoms and time-frequency atoms [6].

\section{The design of cascade atoms dictionary and parameter estimation of HFM signals}

\section{The estimation of $t_{0}$}

Step1, establish the time scale atomic dictionary. Considering the launch signal $\mu(\mathrm{t})$ :

$$
\mu(\mathrm{t})=\frac{1}{\sqrt{\mathrm{T}}} \operatorname{rect}\left(\frac{\mathrm{t}}{\mathrm{T}}\right) * \exp \left\{-\mathrm{j}\left[2 \pi \mathrm{K} \ln \left(1-\frac{\mathrm{t}}{\mathrm{t}_{0}}\right)\right]\right\}
$$

A complete atom dictionary can be expressed as follows.

$$
G_{f}\left\{g_{r}\right\}=\left(\begin{array}{ccc}
g_{r}\left(s_{1}, \tau_{1}\right) & \ldots & g_{r}\left(s_{1}, \tau_{m}\right) \\
\vdots & \ddots & \vdots \\
g_{r}\left(s_{n}, \tau_{n}\right) & \cdots & g_{r}\left(s_{n}, \tau_{m}\right)
\end{array}\right)
$$

where $\tau_{0}$ is time delay parameters, $s_{0}$ is scale parameter. $m, n$ is the collection of $\tau_{0}, s_{0}$. $g_{k}=\sqrt{\frac{1}{s_{k}}} f\left(\frac{1}{s_{k}}\left(\mathrm{t}-\tau_{k}\right)\right)$

Step 2: decompose the signal in a complete atom dictionary. Decompose the received signal in a complete atom dictionary. The signal appears an optimal matching on a component of a complete atom dictionary

$$
a=\frac{1}{s_{0}}-\frac{1}{t_{0}} \cdot\left(\mathrm{b}-\tau_{0}\right)
$$

Step3: detect straight lines through the radon transform. The radon transform of continuous function is as follows [7]:

$$
U(\tau, \rho)=\mathrm{R}[\mathrm{d}(\mathrm{x}, \mathrm{t})]=\int d[\mathrm{x}, \tau+\rho \mathrm{g}(\mathrm{x})] d x
$$

Under the background of the ideal, the peak is shown in points $\left(a_{0}, b_{0}\right)$,

$$
t_{0}=-\frac{1}{20 \tan b} \quad a_{0}=\frac{1}{\sqrt{1+t_{0}^{2}}} \cdot\left(\frac{t_{0}}{s_{0}}+\tau_{0}\right)
$$

\section{The estimation of parameter $k$}

The parameter $\mathrm{k}$ can be estimated through establishing cascade atom dictionary for we have estimated the parameter ${ }^{t_{0}}$.Firstly, estimating cascade atom dictionary based on parameters ${ }^{0}, k$ [8]. the whole number of atom in the dictionary isM $\times \mathrm{N}$.

$$
H_{f}\left\{g_{d}\right\}=\left(\begin{array}{ccc}
g_{r}\left(s_{1}, \tau_{1}\right) & \cdots & g_{r}\left(s_{1}, \tau_{m}\right) \\
\vdots & \ddots & \vdots \\
g_{r}\left(s_{n}, \tau_{1}\right) & \cdots & g_{r}\left(s_{n}, \tau_{m}\right)
\end{array}\right) \square\left(\begin{array}{ccc}
g_{r}\left(t_{0_{1}}, k_{1}\right) & \cdots & g_{r}\left(t_{0_{1}}, k_{m}\right) \\
\vdots & \ddots & \vdots \\
g_{r}\left(t_{0_{n}}, k_{1}\right) & \cdots & g_{r}\left(t_{0_{n}}, k_{m}\right)
\end{array}\right)
$$

The parameter $t_{0}$ has been estimated, then extracting a certain line according to $t_{0}$.Then the maximum matching will be found out which can extract $k$. 


\section{The simulation}

Considering a HFM signal with rectangle envelop,

$\mathrm{u}(\mathrm{t})=\sqrt{20} \times \operatorname{rect}(20 \mathrm{t}) \mathrm{e}^{-\mathrm{j}[2 \pi \times 2770 \times \ln (1-7 \mathrm{t})]}$

The parameter of channel: white gauss noise $R=-10$, the maximum frequency deviation $f d=5$; the power decline $A=-10$; the time delay $S=10$.

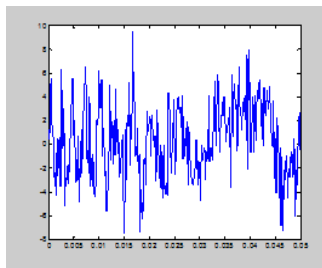

(a)

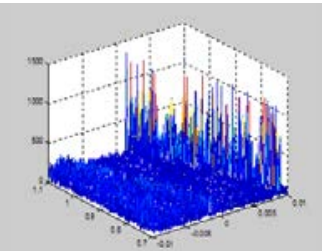

(b)

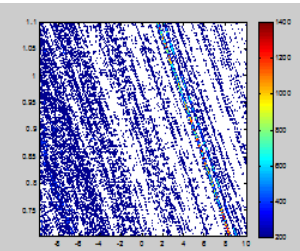

(c)

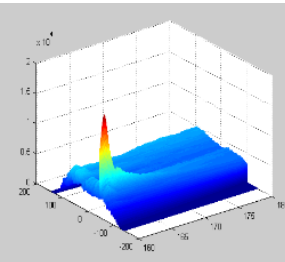

(d)

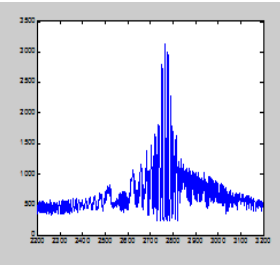

(e)

Figure1:(a): the received signal (b): decomposing the signal (c) extracting the optimal matching (d) extracting the peak through radon transform(e):the estimation of parameter $\mathrm{k}$

The parameter can be obtained: $\boldsymbol{t}_{\mathbf{0}}=7.01\left(\boldsymbol{t}_{\mathbf{0}}=7\right), \mathrm{k}=2768(\mathrm{k}=2770)$.

\section{The pool experiment}

In the pool experiment, the sending and receiving transducer is in a pool of $5 * 15$; the signal sampling rate is $6 \mathrm{KHz}$, the center of the signal frequency is $11 \mathrm{KHz}$.

Considering a HFM signal with rectangle envelop:

$$
\mathrm{u}(\mathrm{t})=\sqrt{20} \times \operatorname{rect}(20 \mathrm{t}) \mathrm{e}^{-\mathrm{j}[2 \pi \times 4770 \times \ln (1-2 \mathrm{t})]}
$$

Through the matlab simulation, the parameters can be got: $1 / t_{0}=2.02, \mathrm{k}=4779$. As the signal is known, and demodulation signal is within the error range, so it can be seen as that the method is feasible.
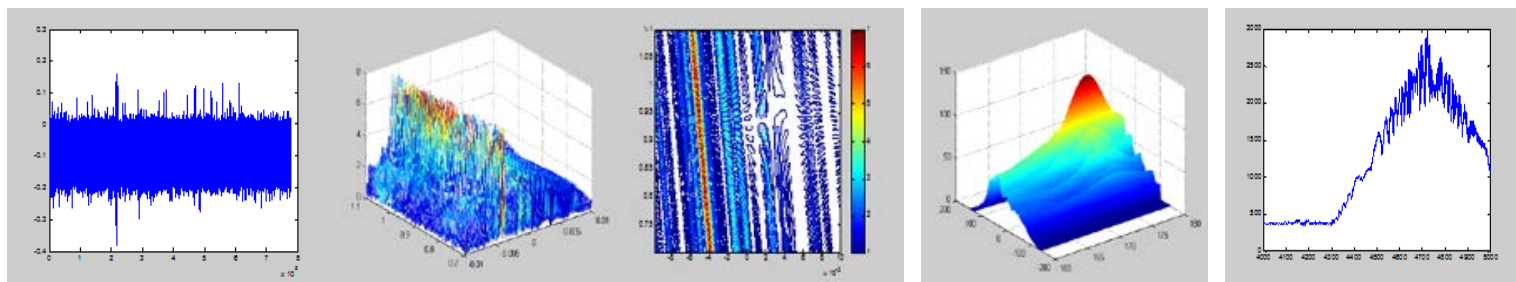

Figure 2:the received signal. Figure3: decomposing the received signal. Figure 4: extracting the optimal matching. Figure5: extracting the parameter $t_{0}$.Figure 6: extract parameter $\mathrm{k}$ of HFM signal

\section{The conclusion}

In this paper, using the HFM signals as launching signal, the cascade atom dictionary is proposed according to the properties of the HFM signal to estimate the parameter of HFM signals. The simulation results and pool experiments show that it is feasible by using cascade atom dictionary to estimate the parameter of HFM signal.

\section{Acknowledgement}

Foundation item: This work was supported by the Fujian Natural Science Foundation of China (2013J01258), the key project in Fujian Province(2012H1012), the National Natural Science Foundation of China(61471309,61107023), Ph.D. Programs Foundation of Ministry of Education of China (20110121120020), the Fundamental Research Funds for the Central Universities (2013121023). 


\section{References}

[1] Kuai X, Sun H, Shen W, et al. Peak-to-average power ratio reduction in underwater acoustic CE-OFDM system by phase modulation[J]. Journal of Convergence Information Technology, 2012, 7(9): 380-386.

[2] YANG J, SARKARTK.Doppler-invariant property of hyperbolic; frequency modulated waveforms[J]. Microwave and Optical Technology Letters, 2006,48 (6):1174-1179.

[3] Lan Zhang, XiaomeiXu: HFM Spread Spectrum Modulation Scheme in Shallow Water Acoustic Channels[J].Oceans, 2012.

[4] Besson, O.; Giannakis, G.B.; Gini, F. Improved estimation of hyperbolic frequency modulated Chirp signals. Signal Processing, IEEE Transactions on, vol.47, no.5, pp.1384-1388, May 1999

[5] Fiorillo A S, D'Angelo G. Echo signals processing with neural network in bat-like sonars based on PVDF[C]//Ultrasonics Symposium, 2002. Proceedings. 2002 IEEE. IEEE, 2002, 1: 781-784.

[6] Analysis of Self-CWT Ridge of Wideband HFM Signal[J]. Journal of System Simulation, 2008,20(19).

[7] F. Hjouj, D. W. Kammler, "Identification of reflected, scaled, translated, and rotated objects from their radon projections,” IEEE Transaction on Image Processing, vol. 17, 2008, pp. 301-310.

[8] Zhang G X. Time-Frequency Atom Decomposition with Quantum-Inspired Evolutionary Algorithms [J]. Circuits, Systems and Signal Processing.2009, 29 (2): 209-233. 